\title{
INDOOR LOCALIZATION WITH PROBABILITY DENSITY FUNCTIONS BASED ON BLUETOOTH
}

\author{
Kai Wendlandt, Marcus Berbig, and Patrick Robertson \\ German Aerospace Center (DLR), Institute of Communications and Navigation, \\ Oberpfaffenhofen, 82234 Wessling, Germany, Kai.Wendlandt@dlr.de
}

\begin{abstract}
We present a simple system to help people navigate inside of buildings or even in outside areas close to buildings. It is based on the "RSSI" and "Transmit power" data of an established Bluetooth link. The system is in principle sufficient for the intended application (pedestrian, indoor), but it is certainly not a high resolution indoor location system. The achievable accuracy is dependent on the setup (number of access points and their constellation and available Bluetooth devices) but will not be better than in the order of several meters. A main feature of this fingerprinting-like system is the representation of the estimated position in the form of probability density functions.
\end{abstract}

\section{INTRODUCTION}

As navigation systems and GPS devices become more and more popular, the need arises to use navigation even in indoor scenarios. But due to the technical constraints of GPS and other global satellite navigation systems (GNSS), it is not feasible to use these in buildings without incurring large errors due to shadowing and multi-path disturbance. Systems like SHADE[1] successfully presented a system with inertial measurement units to assist the GPS receiver.

The idea in the presented system is to use the built in Bluetooth chip-sets, now ubiquitous in most communication devices, to help people navigate even in difficult indoor scenarios, where GPS signals are not available or are disturbed through multi-path propagation.

Much research has been carried out on the accuracy and the drawbacks of common positioning techniques like satellite navigation systems. Also, proprietary location systems have been developed with even higher precision down to only few centimeters[2][3]. The reason for this large variety of localization systems with different accuracies are the numerous applications, which all have different requirements regarding the tolerable positioning error and also on the users dynamics. A car on a highway may cope with perhaps $50 \mathrm{~m}$ accuracy in order to achieve a certain quality-of-service, whereas a system for a pedestrian user looking for a shop in the city is only helpful with $5-10 \mathrm{~m}$ tolerance. But especially this last scenario and other similar indoor applications suffer from multipath reception of the GNSS signals or there is no signal at all. In this case, the accuracy of 5 meters is not achievable[1]. Infrared systems as presented by [4] could help, but have a very limited range and need line of sight for operation.

We therefore focused on a system to operate either completely indoor or in urban canyons to augment GPS/GNSS measurements. The applications we are targeting should enable a person to find the right door on a corridor or to find the right exit in a hotel lobby. This is why our intended system accuracy is about 5 meters and not more accurate.

There are three competing principles[3, pages 78,79$]$ to determine a position for a mobile user - not limited to indoor scenarios. The first one operates with directional antennas at the receivers to determine an angle of arrival (AOA) of a radio transmitter. But there are strong limitations in multipath environments (indoor) due to reflections from wrong directions. The second principle needs specialized hardware at the transmitter and the receiver to determine a distance between two stations. This is can be achieved by measuring the signal delay points (time of arrival, TOA or time difference of arrival between different stations - TDOA). The use of triangulation algorithms then leads to a position when several such stations are in range. In general, this can be the most precise method of location determination depending on the complexity of the setup. The last type of algorithms uses signal strength and other parameters measured from a radio link. The data is used either to directly determine a distance (free-space transmission loss proportional to $1 / r^{2}$ ) or to calibrate a room or an area with typical measurement data. This last method is a kind of fingerprinting system which we intend to implement based on the Bluetooth short range radio system. In comparison to similar systems like[5], we used the combination of probability density functions to determine the position instead of ana-

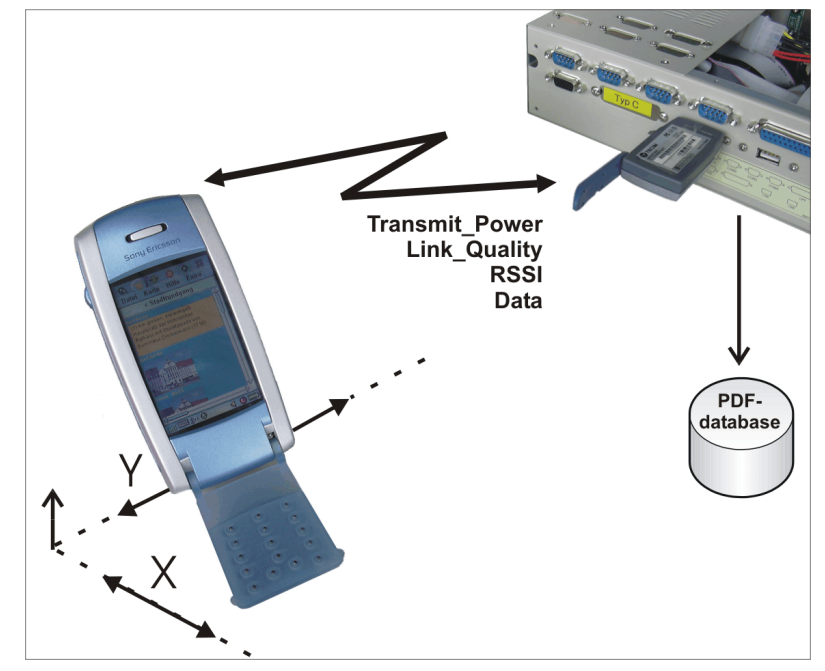

Fig. 1. Measurement setup with mobile device and Bluetooth local service point (LSP). Measurement and positioning data is stored locally and processed offline to build a database of probability density functions (PDFs) 
lyzing the measurement samples with the $k$-nearest-neighbors technique.

Our data representation and interpretation is the main focus of this paper. Obviously a system that relies on measured data needs to run through a calibration process - at least once during an initialization phase. Minimization and interpolation of these calibration measurement is another topic of this publication.

\section{Bluetooth And System Fundamentals}

As described in the previous section, many navigation systems need specialized hardware. Our goal was to not use additional hardware at the mobile terminal. The only wireless short range communication that will be available in mobile phones or other handheld devices (characterized by low power consumption) in the near future seems to be Bluetooth. It was developed to replace (serial) cables between computers and peripherals. But due to its open protocol stack, many new applications were developed like data exchange between mobile phones or personal area networks.

In contrast to the variety at the higher layers, the physical layer is firmly standardized to achieve compatibility between all Bluetooth equipped devices. Importantly, Bluetooth consumes significantly less power than WirelessLAN. One reason for this is the power control of Bluetooth which tries to keep the power at the receiver within the so called golden receive power range. This is a well defined power range between two threshold levels with a dynamic range of $20 \mathrm{~dB}[6]$. The Received Signal Strength Indicator (RSSI) shows the deviation between the measured signal power and the limits of the golden receive power range. Any positive RSSI value indicates how many $\mathrm{dB}$ the signal is above the upper limit, any negative value shows the distance below the lower limit. If the signal is inside the $20 \mathrm{~dB}$ range, the RSSI value is defined to be zero. Besides this value, there is another valuable parameter that can be recorded from the Bluetooth stack: The Transmit_Power_Level indicates the actual output power of the RF front end. The values may range from $-30 \mathrm{dBm}$ to $20 \mathrm{dBm}$. Depending on the power class of the Bluetooth chipset, the real control range can vary according to the specification. The third possible parameter to characterize a Bluetooth link is the Link_Quality. Big changes in this value occur only in bad conditions, when transmitter and receiver are far away from each other and the RSSI is already below the lower limit of the golden receive power range. Moreover, it is up to each chipset manufacturer how they exactly define the link quality. These are the reasons why we considered this parameter to be less important for our investigations. But despite this standardization of levels and ranges, the RSSI values still show a slight variation between different chipsets. Thus the calibration process has to be performed carefully.

In the following, we intend to use the two mentioned parameters (RSSI and Transmit_Power_Level) to compute an Area of Probable Position (AoPP). The reasonable combination of multiple AoPP finally leads to a more precise location estimation[7].

\section{MEASUREMENTS}

Our measurements took place in a laboratory of size $12 \times 7$ meters with two pillars in the middle and another obstacle at the wall opposite the door (see resulting floor plan in measurement results). A grid of $1 \times 1 \mathrm{~m}$ defined the points where measurements were taken. The mobile terminal was placed at a height of $1.40 \mathrm{~m}$ on a non-metallic pylon to prevent possible influences of signal reflections by normal tripods and to simulate in a simple way a person carrying the device, even though it does not model the particular influence of the user's body on the signal path. See figure 1 for the general measurement setup.

The handset used is an off the shelf SonyEricsson P800 Smartphone. This mobile runs Symbian 7.0 UIQ, which is programmable by third party application developers. A special $\mathrm{C}++$ software (denoted as the Bluetooth Adapter) which we have developed handles the connections to the fixed Bluetooth stations. It waits for incoming connections and responds to a L2CAP-connection request of the master of the piconet. The master (i.e. the fixed station) is a small embedded computer with a Tecom BT3030 Bluetooth dongle attached to its USB port. In the following we will refer to it as a "local service point" (LSP). On top of this L2CAP communication link applications on the phone can exchange data with applications on the LSP. This communication infrastructure is part of the Heywow-project[8], which demonstrated tourist oriented location dependent services in the town of Landsberg am Lech, Germany.

At the local service point, we register 10 to 20 samples of the previously introduced RSSI and Transmit_Power_Level

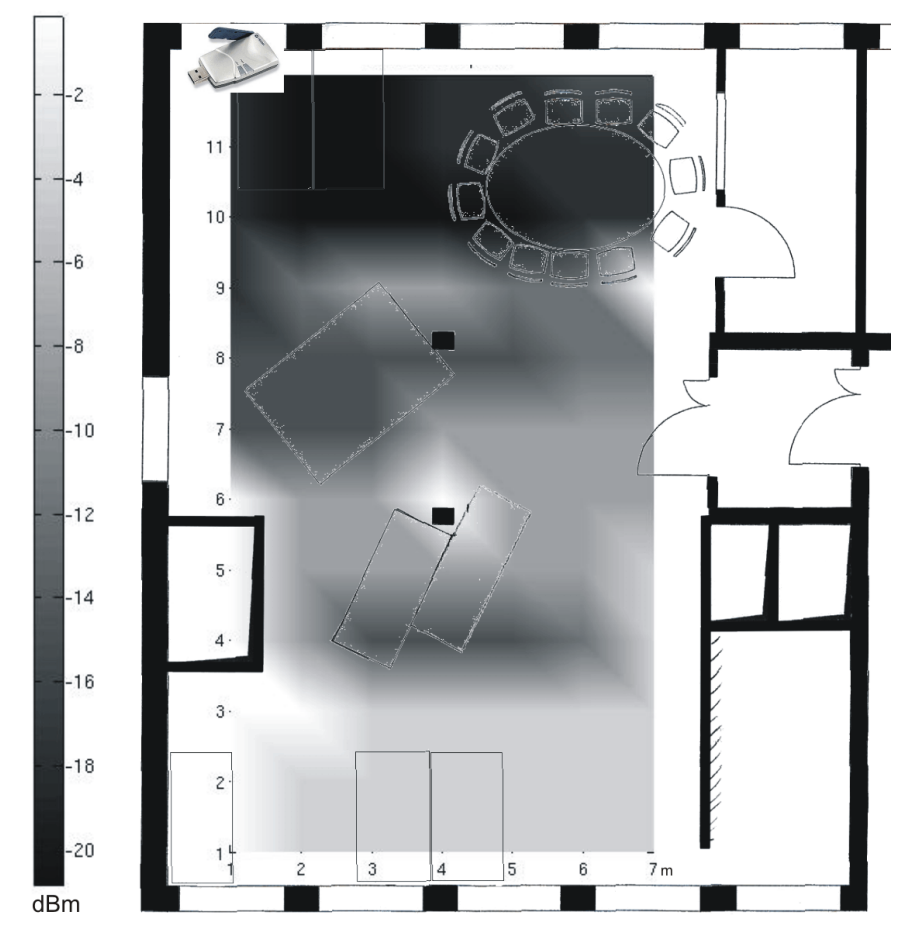

Fig. 2. Transmit power $(\mathrm{dBm})$ of the Bluetooth dongle measured at each position in the room 
data of the established Bluetooth link to our mobile device. The corresponding position data of the mobile in the laboratory was recorded manually. The recording of these samples was repeated for all 80 equally distributed points in the laboratory. The resulting RSSI and Transmit_Power distribution over the whole grid is then further processed to result in probability density functions for each measured value. This process is described in detail in section IV.

The measurements were repeated for two more positions of the LSP in the laboratory. This is part of the earlier mentioned calibration process of our System. The optimized number of measurement points for a room and optimal LSP distribution in the building is subject to ongoing investigations. But it turned out, that two is the minimum number of LSP to result in a reasonable location estimation. This is especially valid for outdoor scenarios, where a person can disturb the free field propagation of the signal. For indoor scenarios, two is also enough but due to multipath propagation, it is better to have three LSPs in range. We also assume that additional location sensors will be employed in addition to radio based systems and that the resulting sensor fusion will lead to sufficient accuracy with only 2-3 LSPs.

The measurements were done in sequence since at this time the phone hardware and firmware does not support multiple concurrent Bluetooth connections to different LSPs.

\section{A. Measurement examples}

The collected data of two parameters from three LSP positions result in six distributions like the one shown in figure 2. The dark color indicates a low transmit power in the area close to the local service point in the upper left corner. Significantly higher power is necessary, if the mobile is behind the obstacle at the lower left wall (light gray). In the middle of the room, the transmit power varies because of changing conditions due to multipath propagation. From all measured data, we can generate probability density functions according to the algorithms described in the next section.

Further measurements in a long corridor and an outdoor area showed similar results and could in future be used to define a simple model based on the distance between transmitter and receiver. This is necessary to reduce the number of calibration points in future practical implementations.

\section{SOFTLOCATION WITH PROBABILITY DENSITY FUNCTIONS}

At present the main question of (self-)localization is usually: "Where am I?" But this can lead to problems because of limitations in the real world: e.g. faulty measurements, bad satellite constellations (DOP) or errors due to multi-path propagation. Real systems are not able to provide the location with infinite accuracy, and they often vary considerably (both short term and long term variation) in the accuracy they achieve. Therefore, it seems to be better to ask another question: "With which probability am I at a specific place, given all my measurement data". Expressing and computing this posterior probability leads to a Bayesian approach. Thus the problem is formulated using conditional probabilities, over discrete random variables, of the form:

$P($ location $\mid$ measurement $)=$ probability of location given a specific observation (measurement)

Using Bayes' Rule, we get:

$$
P(\text { loc } \mid \text { meas })=P(\text { meas } \mid \text { loc }) \frac{P(\text { loc })}{P(\text { meas })}
$$

We assume that the prior probability of the location $P(l o c)$ to be uniform over the set of possible locations - it is equally distributed over the whole laboratory. The measurement data in our trials were the discrete RSSI or Transmit_Power values. The probability of each measured value $P($ meas $)$ can be estimated from a histogram of all measured values. The conditional probabilities $P$ (meas $\mid l o c)$ normally should be determined by a large number of measurements at a fixed location. This way, we would get a fair distribution of the observed measurement values at this specific position, under all manner of circumstances that the actual user will herself experience during actual navigation. In practice, this is not feasible, however. Especially if we consider the fact that calibration measurements are needed for each room in a building, the time needed to calibrate should not exceed certain limits. Hence we decided to derive the required distribution from the eight neighboring measurements around a grid location in the room (figure 3). A more generalized method would be to assume a Gaussian distributions of the two measurement data variables, conditioned on each position, and thus characterized by mean and variance. The first step of the calibration process would be to estimate the values of each mean and variance at each location, thus defining $P($ meas $\mid l o c)$. All RSSI and Transmit_Power data values are computed according to equation (1). The resulting matrices comprise the complete calibration database for the measured area.

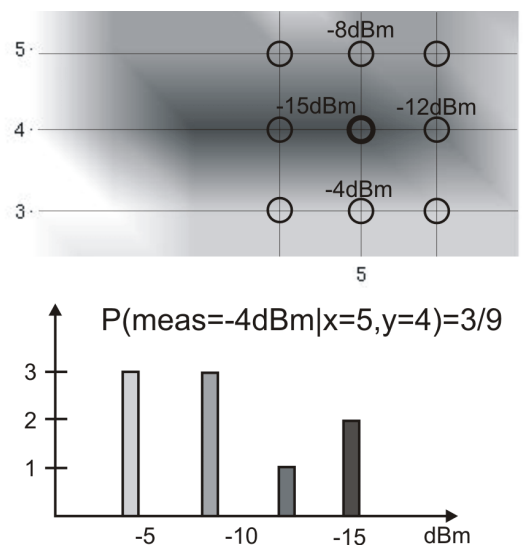

Fig. 3. Example computation of conditional probabilities $P($ meas $\mid l o c)$ for one fixed location

To verify the positioning of a user with our system, we measure, for example, one Transmit_Power value somewhere in the laboratory. From our database, we extract the values for 

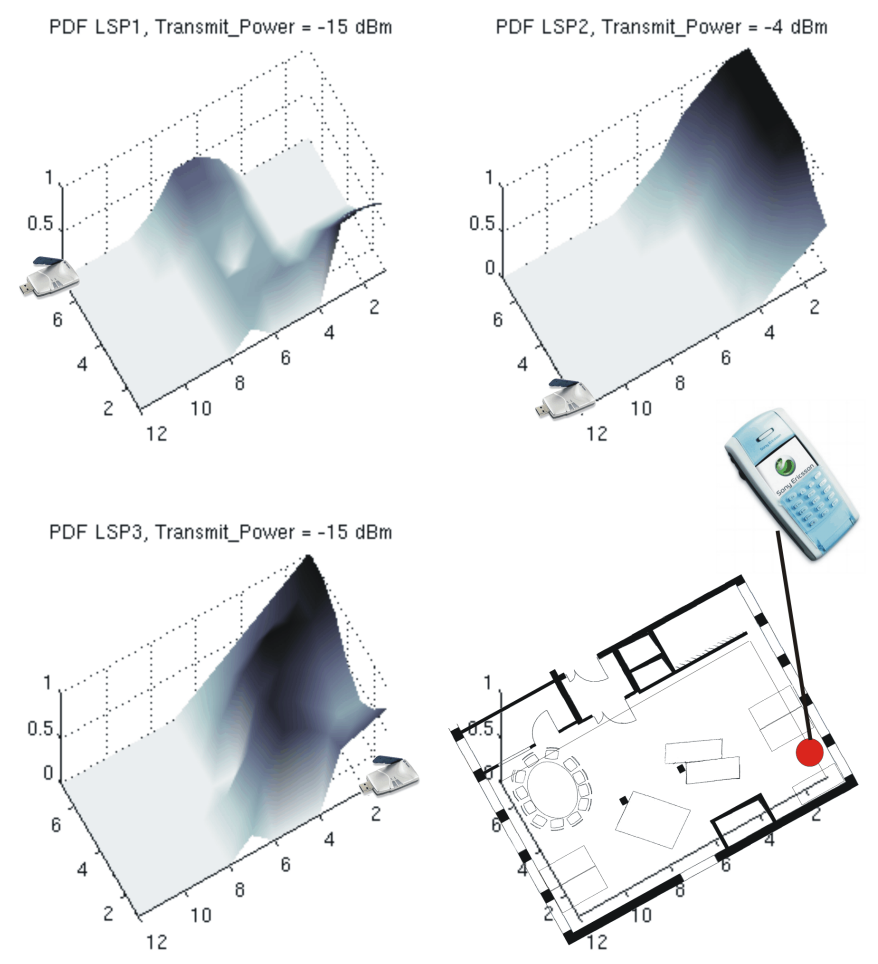

Fig. 4. Probability density functions for different measured Transmit_Power values to three Local Service Points, seen from the same receiver position

$$
P(\text { meas }=\text { measured Transmit_Power } \mid \text { loc })
$$

for each location. Then we normalize each value by

$$
P(\text { meas }=\text { measured Transmit_Power })
$$

to arrive at

$$
P(\text { loc } \mid \text { meas }=\text { measured Transmit_Power }) \text {. }
$$

The resulting posterior distribution expresses our desired area of Probable Position (AoPP). Example distributions for different measured Transmit_Power values from different LSP positions are shown in figure 4. As a single such distribution is not yet very meaningful for position estimation, we simply multiply the distribution by the distributions that result from additional measurements[7] to the remaining local service points in the room or even in neighboring rooms. This assumes independent measurement errors or conditional independence of the measurements meas $s_{A}$ and meas $_{B}$ given a location loc:

$$
P\left(\text { meas }_{A} \mid \text { loc }\right)=P\left(\text { meas }_{A} \mid \text { loc, meas } B\right) \text {. }
$$

The resulting combined distribution (figure 5) gives more accurate position estimation because ambiguities like multiple maxima in the probability density function are usually suppressed as already shown in [9].

We only present probability density functions for the Transmit_Power values, because the RSSI values did not change inside the laboratory. The reason for this is the high output power of the Tecom BT3030 Bluetooth dongle (power class 1 ), so the signal was always inside the golden receive power range. In contrast, with a 3 Com Bluetooth device, the RSSI parameter was more valuable than the Transmit_Power, because this dongle has a shorter range (power class 3 ).

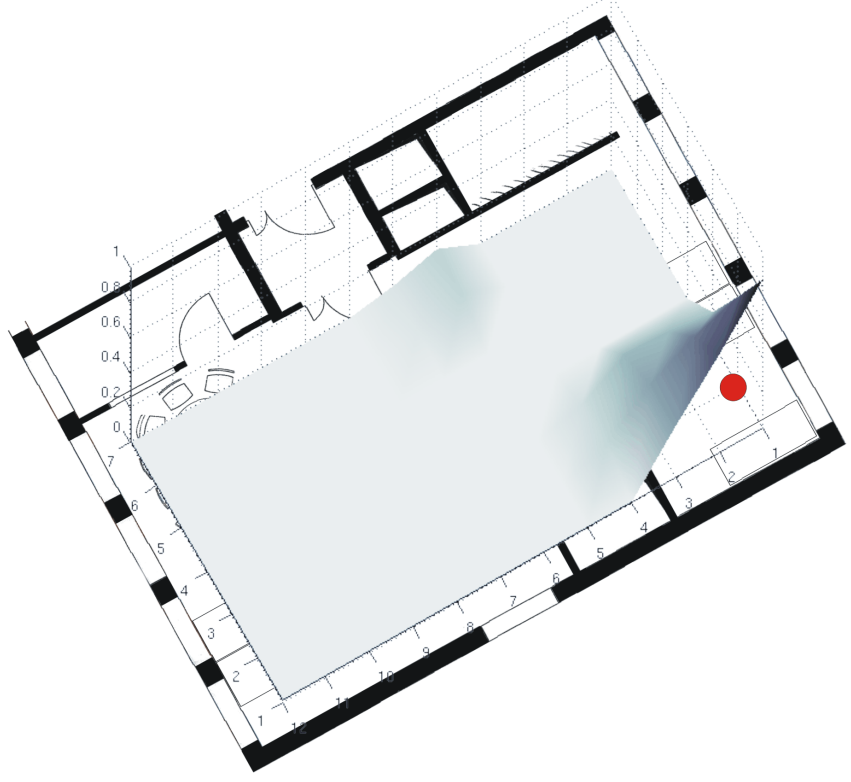

Fig. 5. Combined Probability Density Functions show an Area of Probable Position. The dot at $(2,1)$ shows the position of the measurements (fig. 4)

\section{A. Accuracy}

The achievable accuracy with this method depends on many parameters like the actual distribution of the Bluetooth enabled local service points or the number of obstacles in a room and their dynamics (e.g. other moving persons). The different features of the used Bluetooth chipsets (power classes, Link Quality, Power control) also play an important role. The main problem of a practical system will be the measurement inaccuracy caused by a person carrying the mobile device. Because of all these limiting parameters, we cannot yet conclude a generally valid value for the accuracy. But for our actual setup, the results are presented here.

In our case, the mean distance between real position and estimated position (maximum of combined probability density function) is around $2 \mathrm{~m}$ with a standard deviation of $1.2 \mathrm{~m}$ assuming combination of probability density functions from three local service points. This is the overall mean value for all positions in the room. An example for one position $(x=$ $5, y=9$ ) is shown in figure 6 . If measurements from only two LSPs have been used, the mean distance increased to $2.5 \mathrm{~m}$. The bold circle in figure 6 and 7 shows the mean distance to the real position and the thin circle indicates the standard deviation.

In general, the more sources we combine, the more accurate the result will be, so receiving link parameters from additional LSPs will further improve the accuracy.

All these and future measurements give initial input for evaluations of the positioning accuracy in a Bluetooth equipped area. Thus analysis will be carried out and results will lead to general models which can be used to apply existing indoor channel models for performance analysis. 


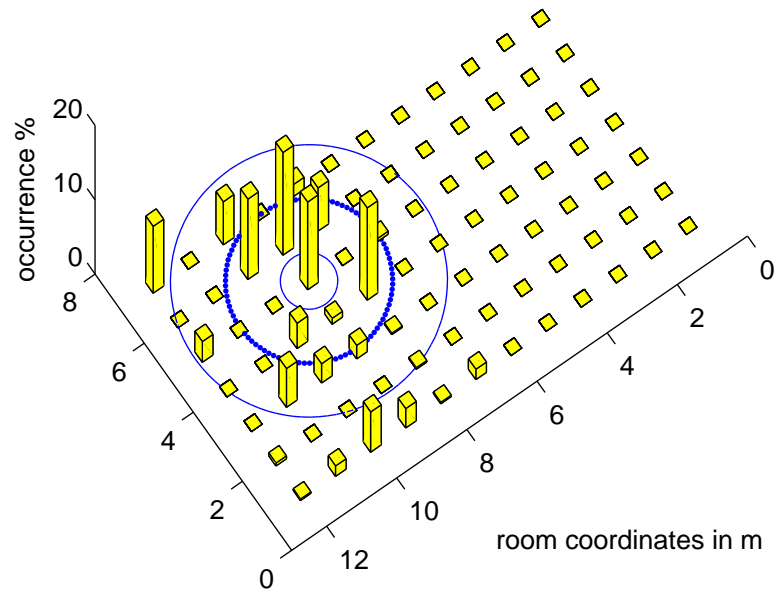

Fig. 6. 3 LSPs: Occurrence of position fixes based on combination of three probability density functions. Random variation of the measurement data (Transmit_Power) around the position $(5,9)$. The mean distance error to the real position is $1.9 \mathrm{~m}$, the standard deviation is $1.2 \mathrm{~m}$

\section{SUMmary}

We presented a simple indoor positioning system with off the shelf Bluetooth equipment and a mobile phone. Based on the parameters of an established data link, the system computes an Area of Probable Position, which can be refined the more such distributions are combined. The intended accuracy for pedestrian navigation in indoor scenarios has been achieved. Mostly, it is even better than $3 \mathrm{~m}$ but it is very much dependent on the actual setup of the local Bluetooth stations and thus the accuracy still has to be validated for other configurations. Work will also continue in the area of verification with other hardware.

Our measurements give initial input to simulations of the positioning accuracy in a Bluetooth equipped area. The measurements show significant dependence on the distance between mobile terminal and the receiver. Because of the so called golden receive power range of Bluetooth, it does not help much to monitor only the RSSI-level. Useful information can be obtained by considering also the transmit power level, as this is the parameter that is controlled by the Bluetooth baseband to obtain a signal strength, which is within the golden receive power range.

A performance analysis with more than one simultaneously connected access point can be carried out when appropriate hardware is available, but the results of this work should apply equally.

Still the effort to profile an area with our method is quite high, but for production systems, a technician could click on its own position on a PDA screen to start measurements at only some characteristic points in the calibration area.

UWB and RFID-Technologies (NFC) are possible short range communications technologies for next generation mobile devices[10]. They can be treated the same way as shown with

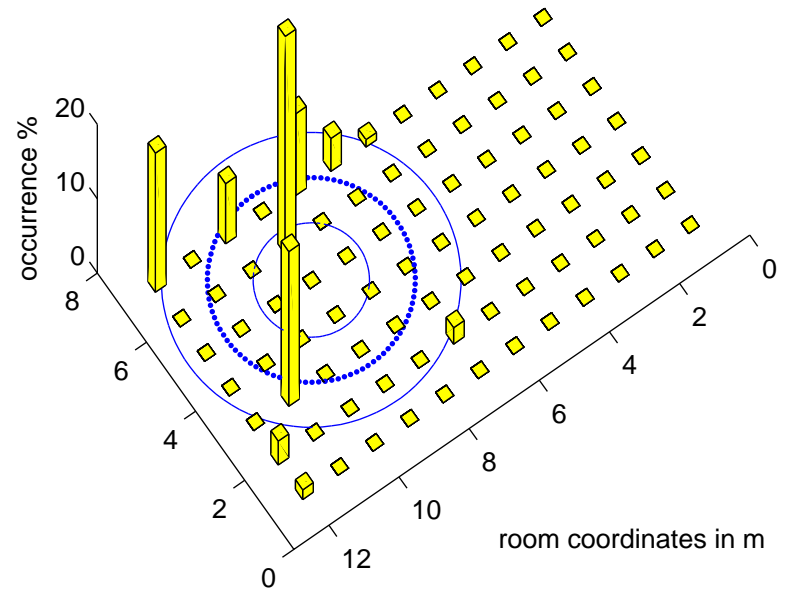

Fig. 7. 2 LSPs: Occurrence of position fixes based on combination of two probability density functions. Mean distance error to the real position is $2.4 \mathrm{~m}$ (bold circle)

Bluetooth if they provide some parameter characterizing the data link. As GPS enabled mobile phones are being introduced into the market, our work can help to achieve a seamless integration from outdoor to indoor navigation.

\section{REFERENCES}

[1] G. Abwerzger, B. Hofmann-Wellenhof, B. Ott, and E. Wasle, "Gps/sbas and additional sensor integration for pedestrian applications in difficult environments," in Institute of Navigation Global Navigation Satelite System - ION GNSS 2004, Long Beach, California, 21-24, 2004.

[2] Fraunhofer IIS. Cairos - a real time and centimetre-precise position measuring system for sport applications. [Online]. Available: http://www.cairos.com/

[3] M. Vossiek, L. Wiebking, P. Gulden, J. Wieghardt, C. Hoffmann, and P. Heide, "Wireless local positioning," IEEE Microwave Magazine, vol. 4, no. 4, pp. 77-86, December 2003.

[4] A. Butz, J. Baus, and A. Krüger, "Augmenting buildings with infrared information," in International Symposium on Augmented Reality, ISAR 2000, IEEE Computer Society Press, 10662 Los Vasqueros Circle, P.O. Box 3014, Los Alamitos, CA 90720-1264, September 2000.

[5] M. Brunato and C. K. Kalló, "Transparent location fingerprinting for wireless services," in Med-Hoc-Net, Mediterranean Wokshop on Ad-hoc Networks, Baia Chia, Cagliari, September 2002.

[6] (2001) Specification of the Bluetooth System, Core, V1.1. [Online]. Available: http://www.bluetooth.com/

[7] M. Angermann, J. Kammann, P. Robertson, A. Steingaß, and T. Strang, "Software representation for heterogeneous location data sources within a probabilistic framework," in International Symposium on Location Based Services for Cellular Users - Locellus 2001, February 2001, pp. $107-118$.

[8] T. Strang, J. Kammann, P. Robertson, M. Angermann, T. Dorsch, C. Wasel, and K. Wendlandt, "Experiences from ramping up an environment for mobile information access," presented at the Workshop on Mobile and Ubiquitous Information Access (MUIA 2004) as part of Mobile HCI '04 - 6th International Conference on Human Computer Interaction with Mobile Devices and Services, Glasgow/Scotland, September 2004

[9] P. Robertson, A. Ouhmich, M. Angermann, and K. Wendlandt, "Implementation of soft location on mobile devices," in International Symposium on Indoor Localisation and Position Finding, InLoc 2002. DGON, Bonn/Germany, July 2002.

[10] M. Tüchler, V. Schwarz, and A. Huber, "Accuracy of an uwb localization system based on a cmos chip," in 1st Ultra-Wideband Expert Talk (UET'05), Niccimon, Hannover, Germany, March 2005. 